\title{
THE BANACH-MAZUR DISTANCE BETWEEN THE TRACE CLASSES $c_{p}^{n}$
}

\author{
NICOLE TOMCZAK-JAEGERMANN
}

ABSTRACT. The Banach-Mazur distance between $l_{2}^{n} \hat{\otimes} l_{2}^{m}$ and $l_{2}^{n} \hat{\otimes} l_{2}^{m}$ is shown to be of the order $\sqrt{\min (n, m)}$. Our proof yields that the distance between the trace classes $c_{p}^{n}$ and $c_{q}^{n}$ is of the same order as $d\left(l_{p}^{n}, l_{q}^{n}\right)$.

In this note we determine the distances between some tensor products of Euclidean spaces $l_{2}^{k}, k=1,2 \ldots$ Let $E, F$ be finite dimensional Banach spaces over the real field. The Banach-Mazur distance $d(E, F)$ is defined as

$$
\inf \left\{\|T\|\left\|T^{-1}\right\| \mid T \text { is an isomorphism from } E \text { onto } F\right\} \text {. }
$$

In this note by $E \hat{\otimes} F$ [resp. $E \hat{\hat{\otimes}} F$ ] we denote the algebraic tensor product $E \otimes F$ endowed with the greatest [resp. the least] norm such that $\|e \otimes f\|=$ $\|e\|\|f\|$ for $e \in E, f \in F$. The space $l_{2}^{n} \otimes l_{2}^{m}$ with the norm

$$
\left\|\sum_{i j} a_{i j} e_{i} \otimes f_{j}\right\|=\left(\sum_{i j}\left|a_{i j}\right|^{2}\right)^{1 / 2},
$$

where $\left\{e_{1}, \ldots, e_{n}\right\},\left\{f_{1}, \ldots, f_{m}\right\}$ are orthonormal bases for $l_{2}^{n}$ and $l_{2}^{m}$ respectively, is denoted by $\mathrm{HS}\left(l_{2}^{n}, l_{2}^{m}\right)$ or simply $\mathrm{HS}$.

THEOREM 1. Let $n, m$ be positive integers with $n \leqslant m$. Then

$$
(2 \sqrt{e})^{-1} \sqrt{n} \leqslant d\left(l_{2}^{n} \hat{\otimes} l_{2}^{m}, l_{2}^{n} \hat{\otimes} l_{2}^{m}\right) \leqslant 10 \sqrt{n} .
$$

Proof. We begin with the upper estimate of the distance $d\left(l_{2}^{n} \hat{\otimes} l_{2}^{m}, l_{2}^{n} \hat{\otimes}\right.$ $\left.l_{2}^{m}\right)$. The argument given below works only for $n \geqslant 36$. However if $n<36$ and $i: l_{2}^{n} \hat{\otimes} l_{2}^{m} \rightarrow l_{2}^{n} \hat{\otimes} l_{2}^{m}$ denotes the formal identity map, then $\|i\| \leqslant 1$ and $\left\|i^{-1}\right\| \leqslant n \leqslant 10 \sqrt{n}$.

We shall construct the isomorphism $T: l_{2}^{n} \hat{\otimes} l_{2}^{m} \rightarrow l_{2}^{n} \hat{\otimes} l_{2}^{m}$ in the form $T=j^{*} \circ u \circ j$ where $j: l_{2}^{n} \hat{\otimes} l_{2}^{m} \rightarrow \operatorname{HS}\left(l_{2}^{n}, l_{2}^{m}\right)$ is the natural embedding and $u$ is an isometry of the $n m$-dimensional Hilbert space $\mathrm{HS}\left(l_{2}^{n}, l_{2}^{m}\right)$. It is easy to check that $\left\|j^{-1}\right\| \leqslant \sqrt{n}$. Since $\left(j^{*}\right)^{-1}=\left(j^{-1}\right)^{*}$ we obtain

$$
\left\|T^{-1}\right\| \leqslant\left\|j^{-1}\right\| \cdot\left\|u^{-1}\right\| \cdot\left\|\left(j^{*}\right)^{-1}\right\| \leqslant n .
$$

Thus the proof of the upper estimate will be complete if we find a $T$ with $\|T\| \leqslant 10 / \sqrt{n}$. This is done in the following proposition.

Received by the editors January 3, 1978.

AMS (MOS) subject classifications (1970). Primary 46B99.

Key words and phrases. Banach-Mazur distance, trace class, tensor products, cross norms. 
Proposition. Let $\mu$ denote the normalized Haar measure on the group $G$ of all linear isometries of $\mathrm{HS}\left(l_{2}^{n}, l_{2}^{m}\right)$. Assume that $m \geqslant n \geqslant 36$. Then

$$
\mu\left\{u \in G \mid\left\|j^{*} \circ u \circ j\right\|>10 / \sqrt{n}\right\}<1 .
$$

Proof. Observe that the set $E$ of the extreme points of the unit ball in $l_{2}^{n} \hat{\otimes} l_{2}^{m}$ equals $S_{n-1} \times S_{m-1}$ where $S_{k-1}$ denotes the unit sphere in $l_{2}^{k}$. It follows from the duality between $l_{2}^{n} \hat{\otimes} l_{2}^{m}$ and $l_{2}^{n} \hat{\otimes} l_{2}^{m}$ that

$$
\left\|j^{*} \circ u \circ j\right\|=\sup \{|\langle j(z \otimes w), u(j(x \otimes y))\rangle| \mid(x, y),(z, w) \in E\},
$$

where $\langle\cdot, \cdot\rangle$ denotes the inner product in the space $\operatorname{HS}\left(l_{2}^{n}, l_{2}^{m}\right)$. The latter expression is equal to the norm of the 4-linear form $\tilde{u}$ on $l_{2}^{n} \times l_{2}^{m} \times l_{2}^{n} \times l_{2}^{m}$ defined by

$$
\tilde{u}(x, y, z, w)=\langle j(z \otimes w), u(j(x \otimes y))\rangle .
$$

Pick 1/8-nets $N_{1}$ in $S_{n-1}$ and $N_{2}$ in $S_{m-1}$ and let $N=N_{1} \times N_{2} \times N_{1} \times$ $N_{2}$. We may assume that card $N_{1} \leqslant e^{3 n}$ and card $N_{2} \leqslant e^{3 m}$. (This can be proved by a standard comparison of volumes argument, cf. e.g. [1, Lemma 2.4].) Thus card $N \leqslant e^{6 n m} \leqslant e^{12 m}$. Given $(x, y, z, w) \in E \times E$ pick $\xi=$ $\left(x^{\prime}, y^{\prime}, z^{\prime}, w^{\prime}\right) \in N$ such that $\left\|x-x^{\prime}\right\|,\left\|y-y^{\prime}\right\|,\left\|z-z^{\prime}\right\|,\left\|w-w^{\prime}\right\| \leqslant 1 / 8$. One can check easily that

$$
\left|\tilde{u}(x, y, z, w)-\tilde{u}\left(x^{\prime}, y^{\prime}, z^{\prime}, w^{\prime}\right)\right| \leqslant\left(\frac{4}{8}\right)\|\tilde{u}\|,
$$

and hence

$$
\|\tilde{u}\| \leqslant \sup _{\xi \in N}|\tilde{u}(\xi)|+\frac{1}{2}\|\tilde{u}\|
$$

so that

$$
\|\tilde{u}\| \leqslant 2 \sup _{\xi \in N}|\tilde{u}(\xi)| .
$$

Observe that

$$
\begin{aligned}
\{u \in G \mid\|\tilde{u}\|>10 / \sqrt{n}\} & \subseteq\left\{u \in G\left|\sup _{\xi \in N}\right| \tilde{u}(\xi) \mid>5 / \sqrt{n}\right\} \\
& \subseteq \bigcup_{\xi \in N}\{u \in G|| \tilde{u}(\xi) \mid>5 / \sqrt{n}\} .
\end{aligned}
$$

We shall use the estimate

$$
v_{\xi}=\mu\{u \in G|| \tilde{u}(\xi) \mid>a\} \leqslant 4 \exp \left(-a^{2} \cdot n m / 2\right)
$$

valid for any $\xi \in E \times E$ and $0<a \leqslant 5 / 6$.

Let $a=5 / \sqrt{n}$. Since $n \geqslant 36$, we have $a \leqslant 5 / 6$ and hence

$$
\begin{aligned}
\mu\{u \in G \mid\|\tilde{u}\|>10 / \sqrt{n}\} & \leqslant \operatorname{card} N \cdot v_{\xi} \leqslant 4 e^{12 m} e^{-25 m / 2} \\
& =4 e^{-m / 2} \leqslant 4 e^{-18}<1
\end{aligned}
$$

which is the desired estimate. 
To estimate $v_{\xi}$ observe that

$$
v_{\xi}=\lambda\left\{t \in S_{n m-1}|\langle j(z \otimes w), t\rangle|>a\right\},
$$

where $\lambda$ denotes the $(n m-1)$-dimensional normalized Lebesgue measure on the sphere $S_{n m-1}$. By the formula 2.4 in [1] the latter number is smaller than

$$
4 \exp \left(-\left(\sin ^{-1} a\right)^{2} n m / 2\right) \leqslant 4 \exp \left(-a^{2} n m / 2\right) .
$$

(The estimate in [1] requires that $\sin ^{-1} a<1$, but in our case $a \leqslant 1-1 / 6<$ sin 1.) This completes the proof of the proposition.

The lower estimate of $d\left(l_{2}^{n} \hat{\otimes} l_{2}^{m}, l_{2}^{n} \hat{\otimes} l_{2}^{m}\right)$ is obvious if one notes that the cotype 2 constant (cf. e.g. [1]) of the first space is $\leqslant 2 \sqrt{e}$ (cf. [3]) while the second space contains an isometric copy of $l_{\infty}^{n}$, hence its cotype 2 constant is $\geqslant \sqrt{n}$. This completes the proof of Theorem 1 .

In the next theorem $c_{p}^{n}$ denotes the tensor product $l_{2}^{n} \otimes l_{2}^{n}$ with the norm

$$
\|u\|_{p}=\left(\operatorname{trace}\left(u^{*} r\right)^{p / 2}\right)^{1 / p} \text { for } 1 \leqslant p<\infty
$$

and

$$
\|u\|_{\infty}=\|u\|,
$$

where $u \in l_{2}^{n} \otimes l_{2}^{n}$ is regarded as a linear operator in $l_{2}^{n}$. Thus $c_{2}^{n}$ may be identified with $\operatorname{HS}\left(l_{2}^{n}, l_{2}^{n}\right)$.

THEOREM 2. For arbitrary $1 \leqslant p \leqslant 2 \leqslant q \leqslant \infty$ one has

$$
(2 \sqrt{e})^{-1} n^{\alpha} \leqslant d\left(c_{p}^{n}, c_{q}^{n}\right) \leqslant 10 n^{\alpha}
$$

where $\alpha=\max (1 / p-1 / 2,1 / 2-1 / q)$.

Proof. Assume that $1 \leqslant q^{*} \leqslant p \leqslant 2$, where $q^{*}=(p-1) / p$. Then $\alpha=$ $1 / 2-1 / q$. We shall prove that $d\left(c_{p^{*}}^{n}, c_{q}^{n}\right) \leqslant 10^{2 / p-1} n^{\alpha}$ and $d\left(c_{p}^{n}, c_{q}^{n}\right) \geqslant(2 \sqrt{e})^{-1} n^{\alpha}$. The remaining cases follow from the identity

$$
d\left(c_{p}^{n}, c_{q}^{n}\right)=d\left(\left(c_{p}^{n}\right)^{*},\left(c_{q}^{n}\right)^{*}\right)=d\left(c_{p^{*}}^{n}, c_{q^{*}}^{n}\right)
$$

and the observation that $2 / p-1 \leqslant 1$.

Let us estimate the norm $\left\|T: c_{q^{*}}^{n} \rightarrow c_{p^{*}}^{n}\right\|$, where $T$ is the operator we have used in the proof of Theorem 1 . We have

$$
\begin{aligned}
\left\|T: c_{q^{*}}^{n} \rightarrow c_{p^{*}}^{n}\right\| & \leqslant\left\|T: c_{p}^{n} \rightarrow c_{p^{*}}^{n}\right\| \\
& \leqslant\left\|T: c_{1}^{n} \rightarrow c_{\infty}^{n}\right\|^{2 / p-1}\left\|T: c_{2}^{n} \rightarrow c_{2}^{n}\right\|^{2-2 / p} \\
& \leqslant\left(10 n^{-1 / 2}\right)^{2 / p-1}=10^{2 / p-1} n^{1 / 2-1 / p} .
\end{aligned}
$$

The interpolation theorem we have used in the last estimate can be found e.g. in [3].

On the other hand

$$
\begin{aligned}
\left\|T^{-1}: c_{p^{*}}^{n} \rightarrow c_{q^{*}}^{n}\right\| & \leqslant\left\|\mathrm{id}: c_{p^{*}}^{n} \rightarrow c_{2}^{n}\right\| \cdot\left\|u^{-1}: c_{2}^{n} \rightarrow c_{2}^{n}\right\| \cdot\left\|\mathrm{id}: c_{2}^{n} \rightarrow c_{q^{*}}^{n}\right\| \\
& \leqslant n^{1 / p-1 / 2} \cdot 1 \cdot n^{1 / 2-1 / q} .
\end{aligned}
$$


Thus we get

$$
d\left(c_{p^{*}}^{n}, c_{q^{*}}^{n}\right) \leqslant\|T\| \cdot\left\|T^{-1}\right\| \leqslant 10^{2 / p-1} n^{\alpha} .
$$

The lower estimate of $d\left(c_{p}^{n}, c_{q}^{n}\right)$ uses the facts that the cotype 2 constant of $c_{p}^{n}$ is $\leqslant 2 \sqrt{e}$ (cf. [3]) and the cotype 2 constant of $c_{q}^{n}$ is $\geqslant n^{\alpha}$. (The latter space contains an isometric copy of $l_{q}^{n}$.)

REMARK. It is well known that if either $1 \leqslant p, q \leqslant 2$ or $2 \leqslant p, q \leqslant \infty$, then

$$
d\left(c_{p}^{n}, c_{q}^{n}\right)=n^{\beta} \quad \text { where } \beta=|1 / p-1 / q| .
$$

The result mentioned in the abstract follows by comparing our results with those in [2].

The author would like to thank Professor T. Figiel for his help in preparing this note.

\section{REFERENCES}

1. T. Figiel, J. Lindenstrauss and V. Milman, The dimension of almost spherical section of convex bodies, Acta Math. 139 (1977), 52-94. (Russian)

2. V. Gurari, M. Kadec and V. Macaev, On the distance between finite dimensional $l_{p}$ spaces, Mat. Sb. 70 (1966), 481-489.

3. N. Tomczak-Jaegermann, The moduli of smoothness and convexity and the Rademacher averages of trace classes $S_{p}$, Studia Math. 50 (1974), 163-182.

Institute of Mathematics, Warsaw University, Warsaw, Poland

Current address: Institute of Mathematics, Warsaw University, PKiN, 00901 Warsaw, Poland 